\title{
Medical Image of the Week: Dual Primary Lung Cancers
}

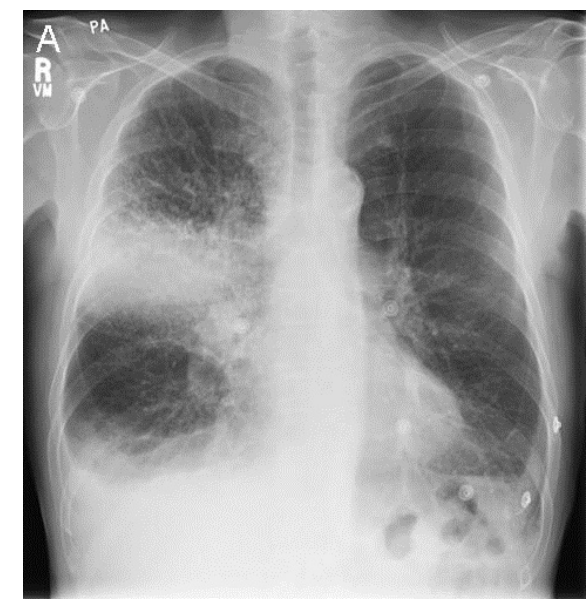

Figure $1 \mathrm{~A}$. Chest $\mathrm{x}$-ray showing mass-like consolidation of the right upper lobe.

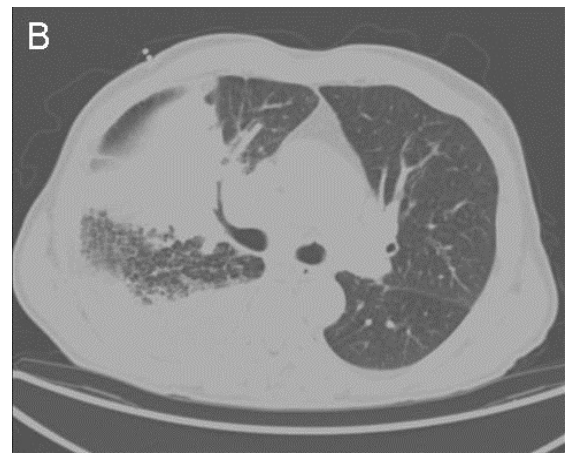

Figure 1B. Thoracic CT showing abrupt cutoff of the apical and posterior segments of the right upper lobe bronchus and encasement of the anterior segment.

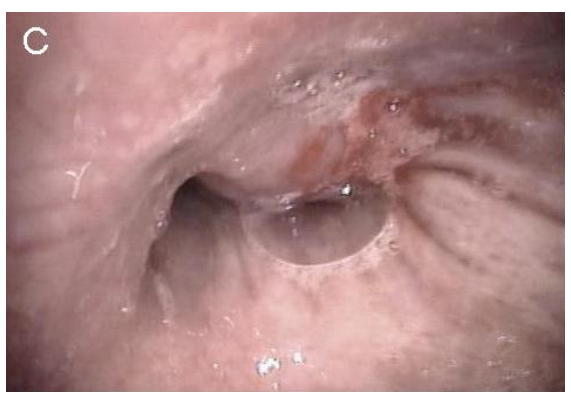

Figure 1C. Endobronchial mass with obstruction of the right upper lobe bronchus. 


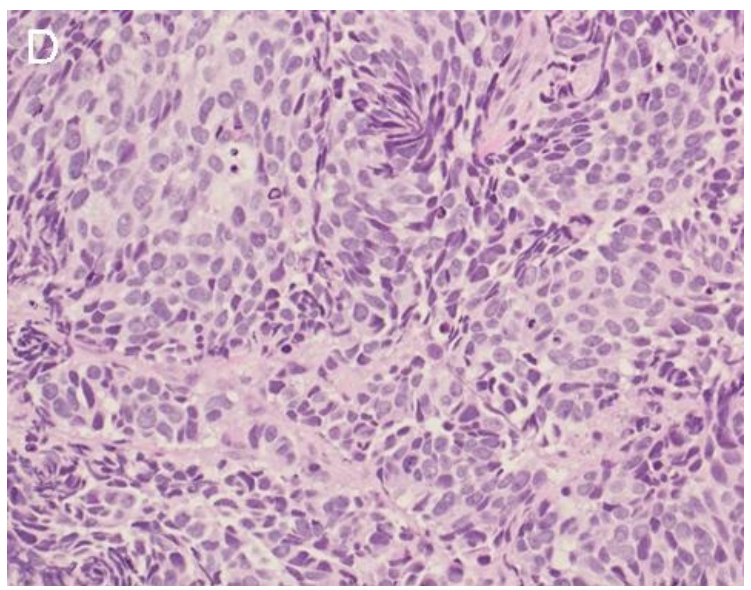

Figure 1D. H\&E stain of right upper lobe mass consistent with small cell lung cancer.

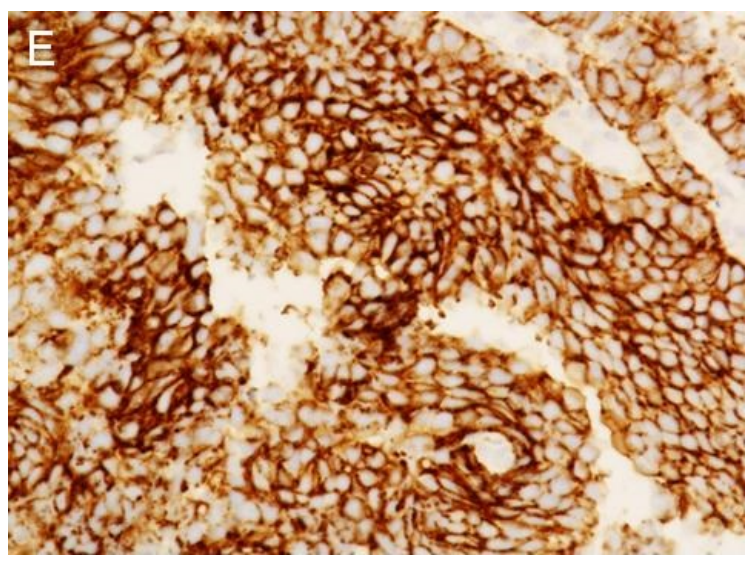

Figure 1E. Positive CD56 staining of the right upper lobe mass.

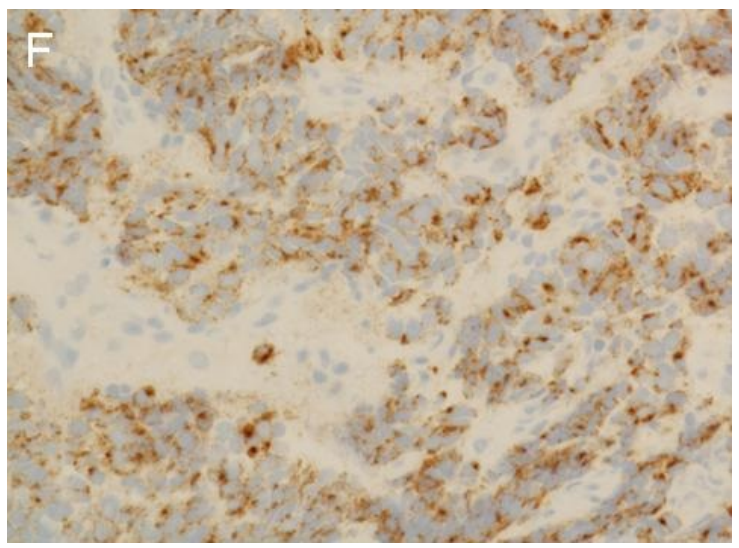

Figure 1F. Positive chromogranin staining of the right upper lobe mass. 


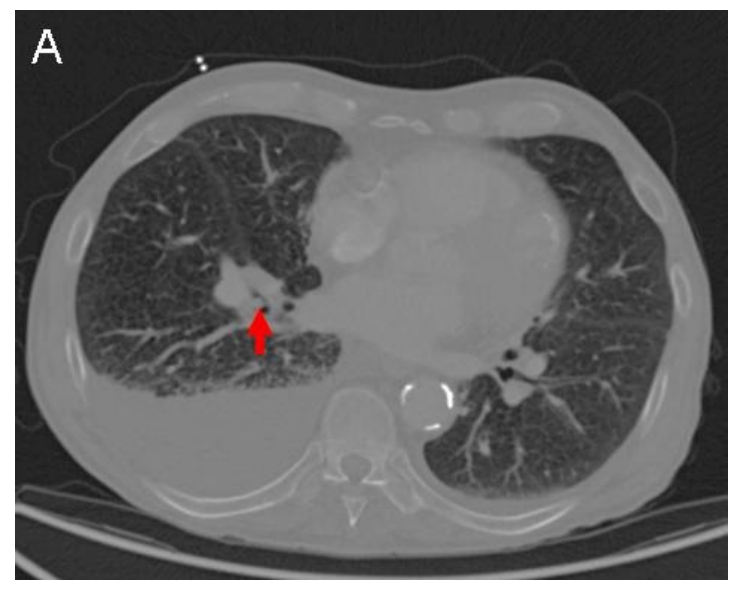

Figure 2A. Thoracic CT showing second lesion (red arrow) at the right lower lobe lateral segment bifurcation.

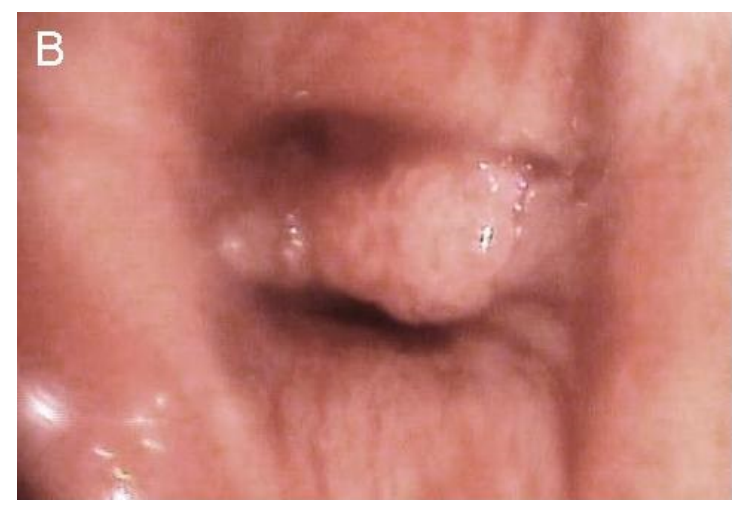

Figure 2B. Endobronchial mass at the lateral segment of the right lower lobe.

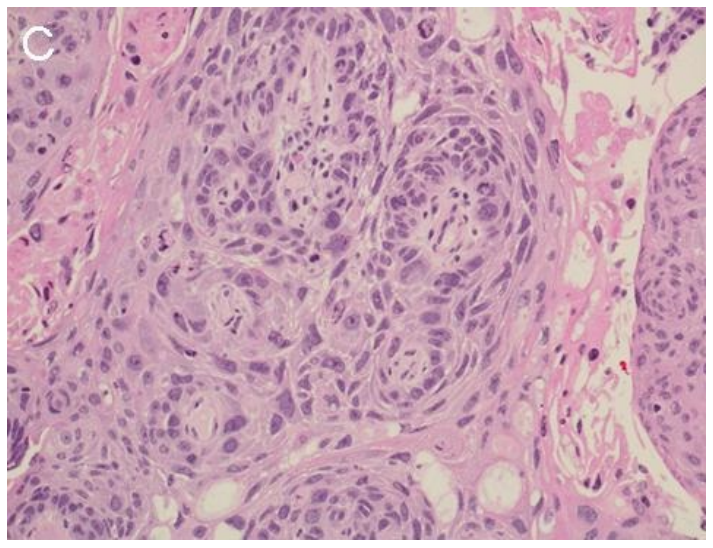

Figure 2C. H\&E stain of right lower lobe mass consistent with squamous cell carcinoma. 
A 73 year old man was admitted to the hospital with complaints of right upper quadrant pain and was found to have consolidation in the right upper lobe (Figure $1 \mathrm{~A}$ ). He was started on antibiotics but failed to have any improvement. A chest CT scan showed a mass-like consolidation with possible mass at the right upper lobe bronchus (Figure 1B). Pulmonary was consulted for bronchoscopy which revealed an endobronchial lesion at the right upper lobe takeoff (Figure 1C) as well as a second endobronchial lesion at the right lower lobe (Figures 2A and 2B). Pathology of the right upper lobe lesion was consistent with small cell carcinoma (Figures 1D-F) while histology for the right lower lobe lesion showed squamous cell carcinoma (Figure 2C). CD56 and chromogranin are important stains used to diagnose small cell lung cancer (1). Data is scarce regarding "synchronous" primary tumors. Though not applicable to our patient, it is estimated that the incidence of dual primary lung cancers is around 16 percent in a patient whose first tumor was surgically resected (2). Our patient opted for hospice care in light of multiple metastases to the brain and abdomen.

Candy Wong, MD; Nathaniel Reyes, MD; Andrea McGonigle, MD; Tan Nguyen, MD; Margaret Rennals, MD; Wei Shen, MD

Department of Pathology and Department of Medicine University of Arizona and Southern Arizona VA Health Care System Tucson, AZ

\section{References}

1. Kontogianni K, Nicholson AG, Butcher D, Sheppard MN. CD56: a useful tool for the diagnosis of small cell lung carcinomas on biopsies with extensive crush artefact. J Clin Pathol. 2005;58(9):978-80. [CrossRef] [PubMed]

2. Johnson $B E$. Second lung cancers in patients after treatment for an initial lung cancer. J Natl Cancer Inst. 1998;90(18):1335-45. [CrossRef] [PubMed] 